\title{
Economic Efficiency Estimation of Region Industrial Complex Functioning in the Context of Subject Economic Interests' Harmonization of Regional Industrial Policy
}

\author{
Dmitry E. Morkovkin \\ Department of Economic Theory \\ Center for Regional Economics and Interbudgetary Relations \\ Financial University under the Government of the Russian Federation \\ Moscow, Russian Federation \\ E-mail: MorkovkinDE@mail.ru
}

\begin{abstract}
In this article is developed by the author of the method and algorithm of evaluation of efficiency of functioning of industrial complex of the region, tested on the official statistics data on the organizational-economic situation of the industrial complex of the Russian Federation. Proposed modified dynamic criterion of estimation of efficiency of functioning of industrial complex of the region, involving the use of a mathematical apparatus of the rank of statistics, as an instrument that provides the optimal trajectory of the development of region's industrial complex.
\end{abstract}

Keywords-economy; region; industrial complex of the region; methodology; economic efficiency; dynamic criterion; regional industrial policy; harmonization of economic interests

\section{INTRODUCTION}

Modern industrial policy as one of the most important institutions of the market economy in the last few years, after almost complete denial (at the Federal level) with the adoption of the Federal law "On Industrial Policy in the Russian Federation" gradually tales a fair place among the tools that form the state economic strategy.

A balanced regional industrial policy should be based on a comprehensive assessment of the effectiveness and key factors of the basic sectors of the region economic core.

This paper proposes a methodological approach to assessing the economic efficiency of the industrial complex of the region as a regional economic system.

Efficiency as a concept at the mesoeconomical level reflects the qualitative and quantitative performance of territorial industrial systems. At the same time, the versatility of the category "efficiency" as a complex of conditions for the interaction of productive forces and industrial relations does not allow to identify a single criterion for its complex quantitative measurement.
The problem of industrial systems assessing the economic efficiency, despite repeated attempts to solve it for many years, still remains quite relevant. In theoretical terms, the concept of "efficiency" is seen by most economists as the ratio "cost - benefit", and the main controversies relate to the clarification of the concepts "costs", "result" and quantifying evaluation. The solution of these issues is significantly complicated by the processes occurring in regional economic systems, in particular, the emergence and development of certain forms in the relations of distribution of social product by economic entities with various modifications of ownership forms.

In our opinion, the problem of determining the evaluator of economic efficiency of functioning of such an economic system as an industrial complex of the region should be approached not from the point of view of the search indicators of the economic activities as such, but from the point of view of the development of the actual evaluation criteria of the industrial complex of the region due to the following reasons. The more capacious and general these criteria are, the less will be the dependence of the assessment on the level of the analyzed system, its structure and cycle of operation, but the more difficult it is to find a measure of efficiency.

\section{SElection OF Evaluation CRITERIA}

A certain step to the resolution of these problems can be an approach to the criterion formation based on the obvious assumption that any economic process does not exist outside of time. The development of the process also takes place in time. It should be borne in mind that time here is not understood in the conventional view, but in its economic aspect - in what we call economic time and base on the assumption that each economic system operates in its own time.

Based on the above, to evaluate the economic efficiency of the industrial complex of the region, we propose a 
dynamic criterion. It sets the trend of the system development, displaying at each time the state to which the mesoeconomical system should strive. One of the properties that distinguish a dynamic criterion from a static one is the form of its representation in the form of a dynamic standarda vector characterizing the state accepted as a reference, ideal.

This state determines the order or ratio of changes in the parameters of the industrial complex functioning in time. Each of the parameters reflects the process occurring in the economic system in a certain plane, isolated from the structure of the system. As a result, the set of parameters reflects the state of the structure in all time dimensions and at the same time determines the mode in which the system functions optimally. Thus, the dynamic criterion forms a structure that provides an optimal trajectory of the industrial complex development.

The number of parameters included in the criterion and reflecting socio-economic processes occurring in the economic system, in general, is not limited. However, the more of them, the more difficult the interpretation of the result, since the surface extracted from the structure will have a very non-elementary shape. On the other hand, using one or two parameters in the criteria is unlikely to have an acceptable result. Since the mathematical apparatus for processing the dynamic criterion includes rank correlation, the necessary minimum are three parameters of the system. According to experts, the inclusion of more than 5-7 parameters in the criterion does not give the expected increase in the quality of the obtained evaluation due to the high complexity of the analysis of the obtained surfaces. Particular importance is attached to the choice and economic content of those parameters that are included in the standard.

It seems natural that at the level of the regional industrial complex, the dynamic standard should be expressed through the existing indicators of the region's industry functioning. However, neither one nor a set of indicators can reflect the dynamics of the process, as they are inherently static and are only a reflection of the state of the system at a given time. Therefore, to construct a dynamic criterion, it is necessary to consider not the values of the indicators themselves, but the order of their movement. It is possible to identify the movement only by measuring the values of indicators at regular intervals. If the movement in the traditional view refers to the movement of an object in space for a certain time, the economic system exists in space, but it cannot move in it, because it is not a material object. Any economic system is like a virtual object with all the distinctive features inherent in it. Therefore, the movement of such an object is captured through the comparison of its structure at different times. Naturally, the fixation occurs through the structure reflection on the process result occurring in the economic system. Each component of the structure exists in its economic time, so there is a need to measure the results of the process through the obtained parameters. Directly they are not commensurable, as they are in different planes and, accordingly, can have different units of measurement. On the other hand, the resulting parameter values characterize a single process generated by the existing structure. Therefore, they can be reduced to a single axis as follows- transformation into dimensionless quantities, but the latter must preserve the quality of the reflected element of the structure, its movement in its own time. Speed and acceleration can act as such evaluators. Speed characterizes the quantitative aspect of movement, acceleration qualitative.

Indicators of the regional industrial complex functioning as a complex economic system are usually presented in the form of time series. Determining the growth rate of the indicator, we find the speed of its movement, and the pace of the rate allows us to fix the acceleration. Thus, the problem of reducing the indicators on a single axis is solved. Having values of accelerations at each moment of time on all indicators, it is possible to estimate a condition of system structure. If in the dynamic criteria to determine the priorities of temporary changes for each parameter, it remains only to compare the criterion state of the structure and actually existing. Such a comparison is quite simple to make using rank correlation.

Dynamic criterion makes sense to apply in the analysis of trends in the development of the industrial complex. The universal approach to the formation of an evaluation through a dynamic standard does not bind the economic system to any evaluation indicator (group of indicators), but reflects the quality of the system state at each moment of its movement relatively to some ideal state determined by the accepted criterion. The formalization of the dynamic criterion is carried out through the ranking of the functioning indicator system of region industrial complex and allows to quantify the effectiveness of its industrial potential use. The formalization of the dynamic criterion, as well as the approbation of the proposed methodological approach to evaluating the economic efficiency of the regional industrial complex is carried out in the work.

\section{THE ECONOMIC INDICATOR SYSTEM CHOICE}

The final results of the regional economic system functioning can be expressed through a set of different economic indicators that characterize the mode of the system operation.

It seems that at the level of the regional economic system, the evaluation criterion should be expressed through the existing indicators of the industrial regional complex in the official statistics (hereinafter - IRC). However, any volume characteristics of the IRC (the volume of shipped products, the average number of employees, etc.), taken in isolation, can not reflect the dynamics of the economic process, as they are inherently static and are a reflection of the system state at one time or another.

When forming a system of indicators that reflects the economic results of the IRC, it is advisable to take into account the following criteria:

- the set does not include indicators that are defined as derivatives of other indicators included in it. This limitation is due to the fact that the necessary minimum of information must be used to describe the state of the IRC. The excessive data set only burdens 
This property has an important characteristic of motion the model, complicating the process of identifying the basic laws of its functioning;

- the set does not include indicators that do not allow to evaluate the change in the system characteristics or its interaction with the environment in the dynamics. This limitation is natural due to the dynamic nature of economic processes;

- the set can include a variety of indicators, both natural and cost, as their relative value is fixed-the growth rate. This condition is dictated by the possibility of reducing heterogeneous indicators of the IRC on one numerical axis for further analysis and verification;

- the set of indicators is a dynamic system. Based on the requirements of changing priorities, it is possible to model a system of indicators;

- the set may include only such indicators, the method of calculation of which has been unchanged for quite a long time. This is necessary to ensure comparability of changes in the considered indicator for the study period.

\section{DYNAMICS CONSIDERATION AND DYNAMIC STANDARD FORMATION}

The formalization of the evaluation criterion is carried out through the "dynamic standard" formation - the order of indicator movement characterizing the IRC state, adopted as a reference (ideal). It is obvious that at the level of the regional economic system, the dynamic standard should be expressed through the existing indicators of the IRC functioning. But neither one indicator nor a set of indicators, as already noted, can reflect the process dynamics, because the indicators are inherently static and are only a reflection of the system state at a given time. Therefore, to construct a dynamic criterion, it is necessary to consider not the values of the indicators themselves, but the order of their movement in time.

The expert way forms a reference rank series - such order of movement of indicators of IRC functioning in time which would indirectly reflect the highest level of parameter coordination of the current state of IRC to target functions of its development according to the accepted criterion. The essence of the standard ranked list is quite transparent - it characterizes the ideal mode of functioning of the industrial regional complex.

\section{INTEGRATED INDICATOR FORMATION IS CARRIED OUT BY FOLDING THE INDICATORS REFLECTING THE STATE OF THE IRC ON ONE NUMERICAL AXIS}

Earlier it was concluded that for the evaluation formation should not be considered numerical values of indicators, but the order of their movement relatively to the standard (dynamic standard). Indicators can be reduced to one axis as follows-conversion to dimensionless values, while the latter must preserve the quality of the structural reflected element. acceleration, which, unlike speed, characterizes the qualitative rather than quantitative aspect of motion.

Performance indicators of the IRC are usually presented in the form of time series. Determining the inflation rate, we find the speed of its motion, and the growth rate allows you to measure acceleration. Given the fact that the growth rate and acceleration are dimensionless quantities, thus, we solve the problem of reducing heterogeneous indicators on one numerical axis.

Movement acceleration of the separate indicator characterizing one of economic activity aspects, in itself is uninformative. We are interested in the economic system as a whole. Given the acceleration values of the entire set of selected indicators, you can consider their position relatively to each other. To do this, it is advisable to use the ranking method: the indicator with the highest acceleration will receive a rank equal to one, and having the minimum acceleration-a rank equal to $\mathrm{N}$ (N-the number of indicators in the set). Now we have at our disposal not abstract values of acceleration of movement, but acceleration indicator rank sets of actual movement for the period, with the help of which it is possible to obtain a certain qualitative characteristic of the socio-economic process. Each obtained set of ranks describes the state of the structure of the economic system at some point in time within the analyzed period through the transformed IRC functioning indicators. This set of ranks contains both the individual components of the transformed metrics and the relationships between them, which is due to the ranking operation.

\section{Quantitative EVAluation OF THE ECONOMIC SYSTEM STRUCTURE}

There is a problem of quantitative evaluation of the structure state. It seems natural that the formed sets of acceleration ranks of the indicator actual movement over the time period should be put in accordance with some ranked (reference) list. In this case, they can be compared while at least in terms of the compliance degree. The essence of the ranked list is quite transparent - this is the ideal mode of the economic system functioning, which provides the most complete coordination of economic interests that are present in the system.

Comparing the actual rank series with the reference, it is possible to obtain some value that will evaluate the effectiveness of the IRC functioning according to the accepted criterion. This comparison can be made using the rank correlation method.

The proposed methodological approach assumes a certain calculation algorithm, including the following steps ("Fig. $1 ")$ : 


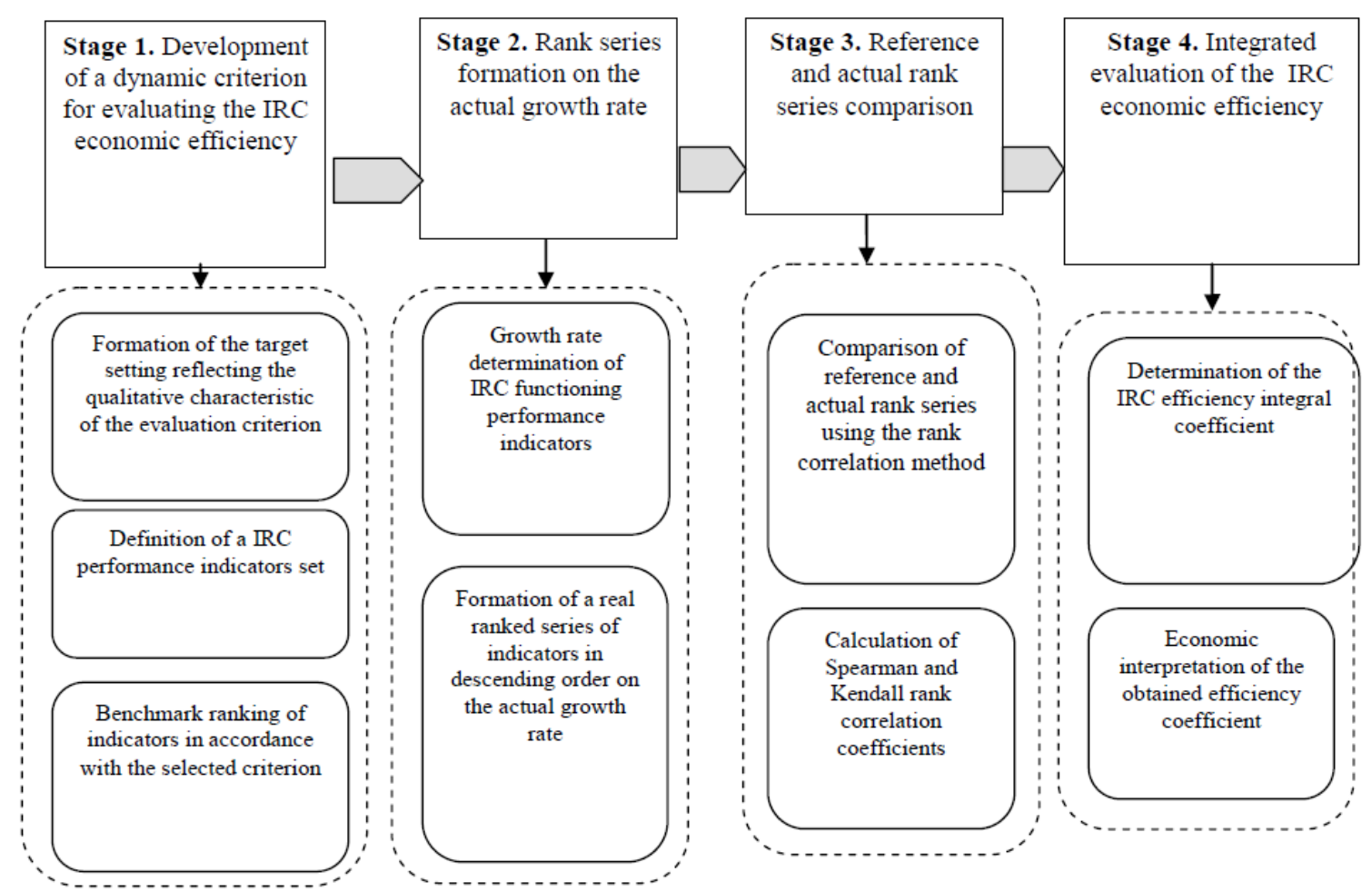

Fig. 1. The Methodology Algorithm for Evaluating the IRC Economic Efficiency.

\section{CONCLUSION}

The introduction and further development of new information technologies can be successfully implemented only when it is the final stage, which is preceded by a whole set of interrelated activities carried out in a strictly defined sequence. The information system of the customs authorities belongs to the class of large and complex dynamic systems. It consists of a large number of elements that implement the managerial functions of foreign economic activity.

The proposed methodological approach to the problem of measuring the economic efficiency of the industrial complex of the region in the economic space of the region, based on the mathematical tools of rank statistics and the use of official regional statistical information, allows to analyze not from the achieved level, but on the basis of evaluating the "distance" to the standard and reduce the indicators of various aspects of management to one scale, which ensures the complexity of the evaluation obtained.

At present, the main problem of industrial policy implementation, both at the federal and regional level, is, in our opinion, the problem of economic interests' harmonization of the state, society and business, i.e. subjects of industrial policy.

It seems to us that it is necessary to conduct a comprehensive study focused on the development of methodology: harmonization of federal and regional industrial policy and harmonization of business economic interests, government and society at the regional level.
The main tool of the study should be a multidimensional model, the parameters of which will be obtained integral quantitative estimates of economic interests in the framework of regional industrial policy. The development of such a multidimensional model will allow to form and model scenarios of regional socio-economic development, to evaluate the results of the development of the region's economy in the implementation of the mechanism of "coordinated" industrial and regional policy at the level of the subject of the Federation.

\section{REFERENCES}

[1] Federal law of 31.12.2014 N 488-FZ (ed. of 03.07.2016) "On Industrial Policy in the Russian Federation "[Electronic resource]. URL: http://www.consultant.ru/document/cons_doc_LAW_173119/ (accessed 11.10.2019).

[2] Yu. A. Friedman, G. N. Rechko, O. A. Biyakov, Yu.Sh Blam. Consistency Level Evaluation of Regional Industrial Policy Subject Economic Interests // Region: Economics and Sociology. - 2008. - No. 3. Pp. $78-96$.

[3] O. A. Biyakov, N. Yu. Kolomarova. Regional Economic Interests and the Problems of Measuring their Coherence. - Kemerovo, 2003. [Electronic resource.] - URL: http://vtit.kuzstu.ru/books/shelf/book5/index.html (date accessed: 11.11.2017).

[4] D. E. Morkovkin. Regional Industrial Complex Development Management on the Basis of the Cluster Approach: monograph / D. E. Morkovkin. - M.: PRIVATE EDUCATIONAL Institution of HIGHER EDUCATION " Moscow University named after S. Yu. WITTE", 2013. - $160 \mathrm{P}$.

[5] D. E. Morkovkin. Methodology for evaluating the efficiency of the regional industrial complex. Economics, taxes \& law - 2011. - No. 6. Pp. 81-94. 


\section{PRESS}

[6] D. Morkovkin, S. Shmanev, L. Shmaneva. Problems and trends of innovative transformation of the Russian economy and infrastructure development // Proceedings of the 3rd International conference on Economics, management, law and education (EMLE 2017) / AEBMR-achievements in the field of business Economics and management, Atlantis Press. 2017. Vol. 32. p. 10-13. DOI: https://doi.org/10.2991/emle-17.2017.3 